ACCEPTED MANUSCRIPT

\title{
Spin preservation during THz orbital pumping of shallow donors in silicon
}

To cite this article before publication: Kamyar Saeedi et al 2019 J. Phys.: Condens. Matter in press https://doi.org/10.1088/1361-648X/ab31d2

\section{Manuscript version: Accepted Manuscript}

Accepted Manuscript is "the version of the article accepted for publication including all changes made as a result of the peer review process, and which may also include the addition to the article by IOP Publishing of a header, an article ID, a cover sheet and/or an 'Accepted Manuscript' watermark, but excluding any other editing, typesetting or other changes made by IOP Publishing and/or its licensors"

This Accepted Manuscript is (c) 2019 IOP Publishing Ltd.

During the embargo period (the 12 month period from the publication of the Version of Record of this article), the Accepted Manuscript is fully protected by copyright and cannot be reused or reposted elsewhere.

As the Version of Record of this article is going to be / has been published on a subscription basis, this Accepted Manuscript is available for reuse under a CC BY-NC-ND 3.0 licence after the 12 month embargo period.

After the embargo period, everyone is permitted to use copy and redistribute this article for non-commercial purposes only, provided that they adhere to all the terms of the licence https://creativecommons.org/licences/by-nc-nd/3.0

Although reasonable endeavours have been taken to obtain all necessary permissions from third parties to include their copyrighted content within this article, their full citation and copyright line may not be present in this Accepted Manuscript version. Before using any content from this article, please refer to the Version of Record on IOPscience once published for full citation and copyright details, as permissions will likely be required. All third party content is fully copyright protected, unless specifically stated otherwise in the figure caption in the Version of Record.

View the article online for updates and enhancements. 


\title{
Spin preservation during THz orbital pumping of shallow donors in silicon
}

\author{
K. Saeedi ${ }^{1}$, N. Stavrias ${ }^{1}$, B. Redlich ${ }^{1}$, A.F.G. van der Meer ${ }^{1}$, R. Mikhaylovskiy ${ }^{2,3}$, A. V. Kimel ${ }^{2}$, \\ C. R. Pidgeon ${ }^{4}$, B. N. Murdin 5
} ${ }^{1}$ FELIX laboratory, Radboud University, Toernooiveld 7, 6525 ED Nijmegen, The Netherlands
${ }^{2}$ Institute for Molecules and Materials, Radboud University, 135 Heyendaalseweg, 6525 AJ Nijmegen, The
Netherlands

${ }^{3}$ Department of Physics, Lancaster University, Lancaster, LA1 1YB, United Kingdom

${ }^{4}$ Institute of Physics and Quantum Science, SUPA, Heriot-Watt University, Edinburgh, EH14 4AS, United Kingdom

${ }^{5}$ Advanced Technology Institute and SEPNet, University of Surrey, Guildford, GU2 7XH, United Kingdom

\section{Abstract}

We investigate the spin relaxation under conditions of optical excitation between the Rydberg orbital states of phosphorus donor impurities in silicon. Here we show that the spin relaxation is less than a few percent, even after multiple excitation/relaxation cycles. The observed high level of spin preservation may be useful for readout cycling or in quantum information schemes where coupling of neighbor qubits is via orbital excitation.

\section{Introduction}

Optical spin pumping of alkali atomic vapor produces a non-equilibrium spin-state through spin selective excitation and spin randomizing relaxation, so that atoms in the selected spin state are gradually depleted through successive excitation/relaxation cycles. This process is crucial for signal enhancement in spin resonance experiments and devices like atomic clocks. Such a technique would be of great interest in the case of shallow silicon impurities, for the state preparation in quantum information technologies [1], and indeed proposals exist for solidstate versions of the atomic clock [2,3]. On the other hand, spin preservation during relaxation will be helpful in quantum gating schemes that involve an excitation and de-excitation cycle [4]. It is crucial for such applications that the spin returns to a definite state ready for the next operation, and in the present work we show that this is indeed true. The lifetime of spins in the ground state is very long at low temperature $[1,5,6]$, so simple thermalization cannot be relied upon to reset the spin [7].

Group V donors in silicon are hydrogenic and similar to the free alkali atoms they have a single spin $1 / 2$ electron loosely bound to a singly positively charged ion. The main difference in the Rydberg excitation spectrum/ of the alkali vapor and the donor is that the high dielectric constant and low effective mass of the donor host produce a much smaller binding energy of a few tens of meV. As the donor is in contact with a solid host whose Debye frequency is also a few tens of meV, there are plenty of vibration modes available giving a phonon emission rate that is much higher than the radiative emission rate. The lower frequency and larger radius (of the orbital states) reduce the spin-orbit (SO) coupling, and there are almost no situations when it is experimentally observable in the Rydberg spectrum above the broadening produced by the phonon relaxation. SO coupling is not visible in the optical excitation even at high magnetic 46 field - only transitions with $\Delta m_{e}=0$ are observed. Those with $\Delta m_{e}= \pm 1$ would be split away from 
the $\Delta m_{e}=0$ peaks by $g \mu_{B} B \equiv 28 \mathrm{GHz} / \mathrm{Tesla}$, but they are not present in the higher resolution Fourier Transform InfraRed (FTIR) spectroscopy [8,9]. Nevertheless, it is interesting to study whether or not the small amount of spin-orbit coupling permits spin randomization during phonon relaxation as in the case of pumping of an atomic clock. In this work we investigate the spin preservation during the excitation-relaxation cycle produced by a resonant Rydberg excitation using a pulsed THz laser, the Free Electron Laser FELIX $[10,11,12,13]$.

The mechanism for spin read-out is resonant creation of donor-bound excitons $\left(D^{0} X\right)[6,14,15]$. Just as the neutral donor $\left(\mathrm{D}^{0}\right)$ is hydrogenic, the exciton $(\mathrm{X})$ is also hydrogenic (positroniumlike), and the $\mathrm{D}^{0} \mathrm{X}$ may be thought of as an analogue of a hydrogen moleeule (or rather positronium hydride). In a magnetic field, the photon energy required for creation of the $\mathrm{D}^{0} \mathrm{X}$ is dependent on the starting spin state of the neutral donor $\left(\mathrm{D}^{0}\right)$ (Fig 1a). The subsequent relaxation pathway may be either by radiative emission or by an Auger process in which the energy produced by the electron-hole recombination is given to the remaining electron, ionizing the donor $\left(\mathrm{D}^{+}\right)$. Because silicon has an indirect band gap, the Auger process dominates and the conduction electrons produced in the reaction $\mathrm{D}^{0}+h v \rightarrow \mathrm{D}^{0} \mathrm{X} \rightarrow \mathrm{D}^{+}+\mathrm{e}^{-}$may be sensed electrically as shown in Fig $1 b$, which is essentially the absorption spectrum. The relative strength of the six $\mathrm{D}^{0} \mathrm{X}$ lines can be used to find the spin polarization. The absorption crosssections for lines $2 \& 5$ are the same, since they are both heavy hole circularly polarized transitions as seen in Fig 1a, and similarly cross-sections for $1 \& 6$ are both circularly polarized light hole transitions, and for $3 \& 4$ are plane polarized light hole transitions. The relative strength of lines 2 and 5 gives the ratio of the spin polarization of the starting states; $L_{5} / L_{2}=u / d$ (and similarly $u / d=L_{3} / L_{4}=L_{1} / L_{6}$ ) where $L_{i}$ of the intensity of /ine $i$ from Fig $1 \mathrm{a}$,b and $u(d)$ is the population of the $m_{e}=+1 / 2(-1 / 2)$ state, i.e. the up(down) neutral donor ground state. This ratio, $u / d$, decreases for increasing magnetic field as shown in Fig $1 \mathrm{~b}$, where the strength $L_{2}$ remains high but the strength of $L_{5}$ rapidly decreases as the up-spin state is depopulated. The rate of change of this ratio $u / d$ is determined by the temperature with a simple Boltzmann factor, as shown in Fig 1c.

(a)

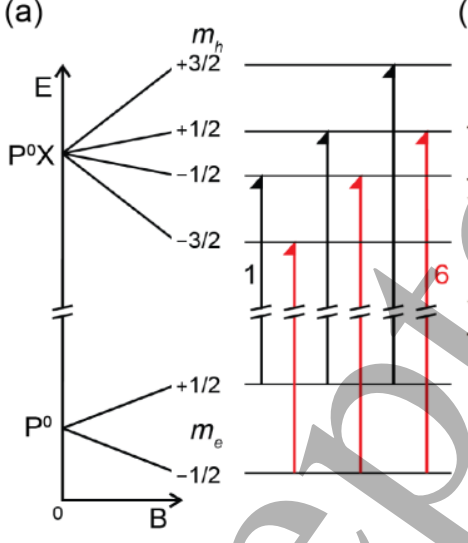

(b)

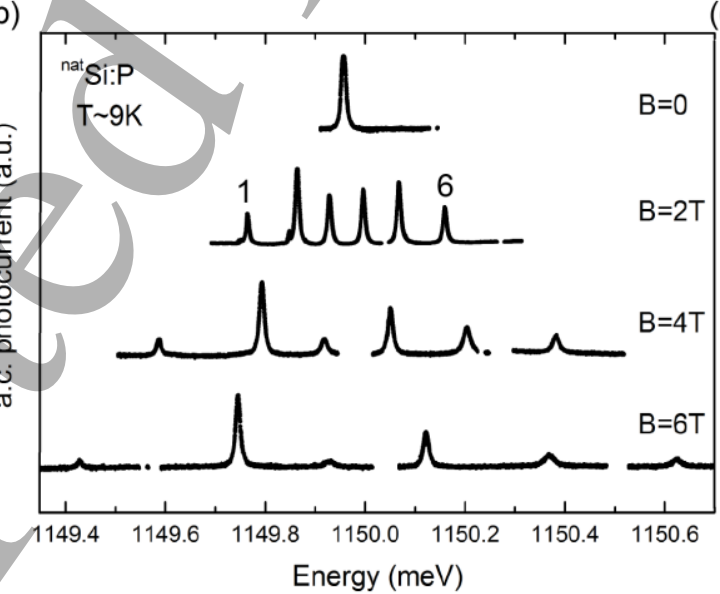

(c)

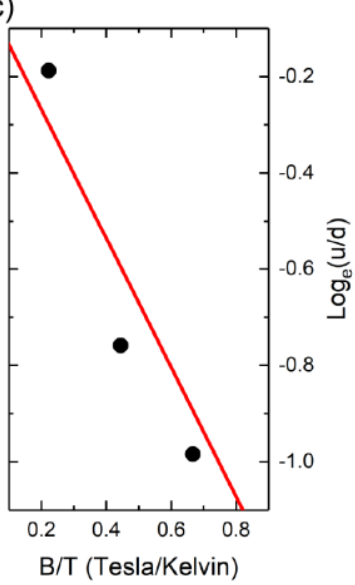

76

78

79

80

81

82

Figure 1. (a) The ground states of the $\mathbf{P}^{0} \mathbf{X}$ and neutral phosphorous $\left(\mathbf{P}^{0}\right)$, and their splittings under a magnetic field, showing the origin of the six electric dipole-allowed absorption transitions, labeled from 1 to 6 in order of increasing energy. The black, odd (red, even) number transitions are from electron up (down) spin. (b) The r.m.s. amplitude spectrum of the a.c. photoconductivity at $T=9 \mathrm{~K}$ for different magnetic fields. (c) The ratio $L_{5} / L_{2}$ where $L_{i}$ of the intensity of line $i$ from (b) (symbols) as a function of B/T. Lines 5 and 2 have the same orbital character (see (a)) and therefore the same matrix element, so $L_{5} / L_{2}=\mathrm{u} / \mathrm{d}\left(=L_{3} / L_{4}=L_{1} / L_{6}\right)$ where $\mathrm{u}(\mathrm{d})$ is the population of 

$g \mu_{B} B / k_{B} T=-1.34 B / T$ (red line).

86

\section{Experiment}

The spin polarization was set initially by a magnetic field and temperature such that $\mu_{B} B>k_{B} T$. The sample in this work, was a commercially sourced float zone silicon, 700 microns thick, with a phosphorus concentration of $[\mathrm{P}] \sim 2 \times 10^{14} \mathrm{~cm}^{-3}$, had a growth direction along $\langle 110\rangle$, two sides cleaved along $\langle 111\rangle$ directions and two sides cut along $\langle 112\rangle$ producing a $5 \times 5.6 \mathrm{~mm}$ square. It was mounted almost strain free between two copper strip in a pouch configuration built on a printed circuit board (PCB) with the magnetic field along the $\langle 112\rangle$ axis. Thermal contact was provided by He exchange gas inside the sample compartment of an Oxford Instruments SpectromagPT cryogen free split-coil magneto-optical superconducting magnet system. The horizontal field magnet system provided optical access to the sample along two axes, a variable magnetic field (up to 7 Tesla) and low temperature environment (down to $1.5 \mathrm{~K})$.

The orbital excitation was provided by THz radiation from the FELIX free electron laser. The macropules arrived at 5 or $10 \mathrm{~Hz}$ repetition frequency, and consisted of few $\mu$ s-long trains of ps long micropulses at $25 \mathrm{MHz}$ repetition frequency. The intensity of the pulse was adjusted via calibrated wire grid attenuators. The pulses are bandwidth-limited [16] and the corresponding FWHM pulse length was 5-9 ps. The average macropulse energy was roughly $250 \mu \mathrm{J}$ in front of cryostat, and the beam was focused onto the sample using a $15 \mathrm{~cm}$ focal length off-axial parabolic mirror (so the spot size was estimated to be about $1 \mathrm{~mm}$ ).

After the excitation-relaxation cycle the spin polarization was read out using $\mathrm{D}^{0} \mathrm{X}$ spectroscopy. The sample mount acts as capacitor plates which allowed measurement of the a.c. response. The a.c. current response to the a.c. bias of $1 \mathrm{~V}$ r.m.s. is modified by the extra electrons produced by ionizing donors (c.f. text above relating to Fig 1b), which produces changes to both the real and imaginary parts of the dielectric response of the crystal. The capacitance is very low, and the a.c. photocurrent is dominated by effects due to the loss term rather than the polarizability term.

The circuit consisted of a Tektronix arbitrary waveform generator (AFG3102) producing the a.c. bias connected to one of the capacitor plates via a coaxial cable, and a FEMTO DHPCA100 transimpedance amplifier measuring the current connected to the other plate. Data was collected using a National Instruments PXIE-5162 digital oscilloscope.

The $\mathrm{D}^{0} \mathrm{X}$ were produced using a CW Toptica Tapered Amplifier (TA Pro100) diode laser operating in the near infrared. The laser frequency was tuned via an external cavity and scanned across the $\mathrm{D}^{0} \mathrm{X}$ resonance by applying a voltage to a piezo attached to the cavity. Spontaneous emission from the tapered amplifier was removed from the spectrum using an angle tunable Semrock long pass filter.

The resonances red-shift with temperature (Fig 2a), though no red-shift was observed due to FELIX laser excitation, proving that the sample average temperature was unaffected by the

laser. Based on the mechanism for spin-lattice relaxation of the donor spins in silicon, $1 / T_{1}$ is 130 proportional to the temperature $T$ and to the fourth power of the static magnetic field [17]. A 131 study of the line widths (Figs 2b, c) shows that the lines are Gaussian in shape and hence 132 inhomogeneously broadened at low temperature. As the temperature is increased, the 
133 homogenous contribution (Lorentzian width) takes over, indicating a temperature-dependent 134 dephasing or population scattering process. The field dependence is in accord with the latter, 135 since the line width increases with the energy of the final state, i.e. the $m_{h}=+3 / 2$ heavy hole 136 which has the largest Zeeman energy (Fig 1a) has the largest width at high field, while the $m_{h}=-$ $1373 / 2$ with lowest excited state Zeeman energy is virtually unaffected by the field. This indicates 138 that the relaxation between the Zeeman-split $\mathrm{D}^{0} \mathrm{X}$ states increases rapidly with increasing $B_{0}$ 139 $\left(1 / T_{1} \sim B_{0}^{4},[17]\right)$. Thus, $\mathrm{D}^{0} \mathrm{Xs}$ created in the higher energy hole Zeeman states can decay rapidly to lower energy $\mathrm{D}^{0} \mathrm{X}$ states as $B_{0}$ increases, but $\mathrm{D}^{0} \mathrm{X}$ s created in the $-3 / 2$ state have no 141 possibility of downwards transitions.

142

(a)

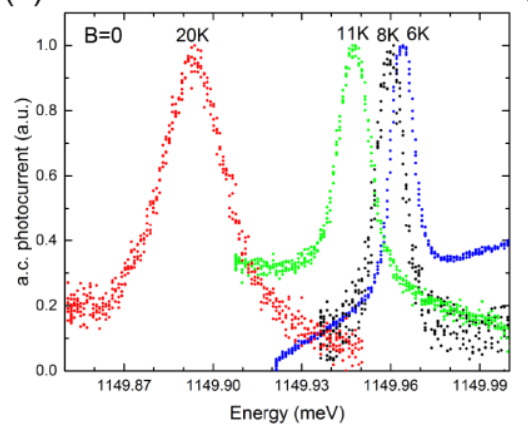

(b)

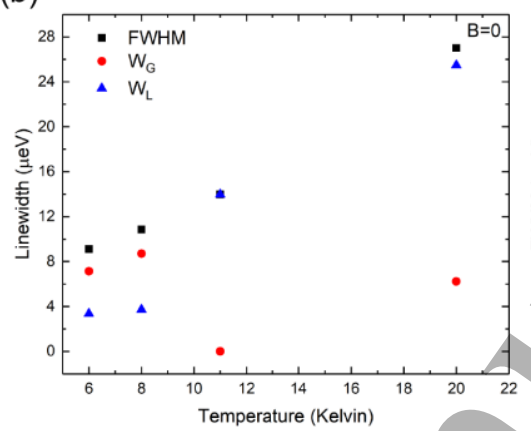

(c)

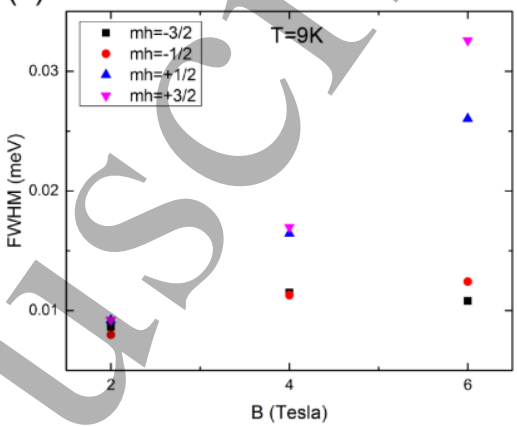

144

145

146

147

148

149

150

151

152

153

154

155

156

157

158

Figure 2. (a) Zero field r.m.s. a.c. photocurrent spectra at $T=6,8,11$ and $20 \mathrm{~K}$ for fixed a.c. bias. (b) Temperature dependence of the full width at half maximum (FWHM) of the $\mathrm{D}^{0} \mathrm{X}$ line (black) based on the Voigt profile fit to the spectra shown in (a) at $B=0 T$. The Gaussian and Lorentzian contributions to the Voigt profile, $w_{G}$ (red), and $w_{L}$ (blue), respectively, show that at high temperature the homogeneous width rises significantly above the inhomogeneous width. (c) Magnetic field dependence of FWHM at $T=9 \mathrm{~K}$ for transitions to different hole spin states from (Fig 1b).

\section{Results}

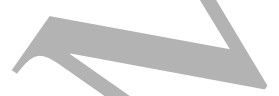

This a.c. measurement circuit includes passive stray capacitances and inductances from the wires etc, and active components such as the source and measurement units. To optimize the sensitivity of the circuit, we measured the effect of the bias frequency on the circuit response to both lasers, shown in Fig 3. The near-IR laser (Toptica) producing the $\mathrm{D}^{0} \mathrm{X}$ produces maximum photocurrent response at about $90 \mathrm{kHz}$.

(a)

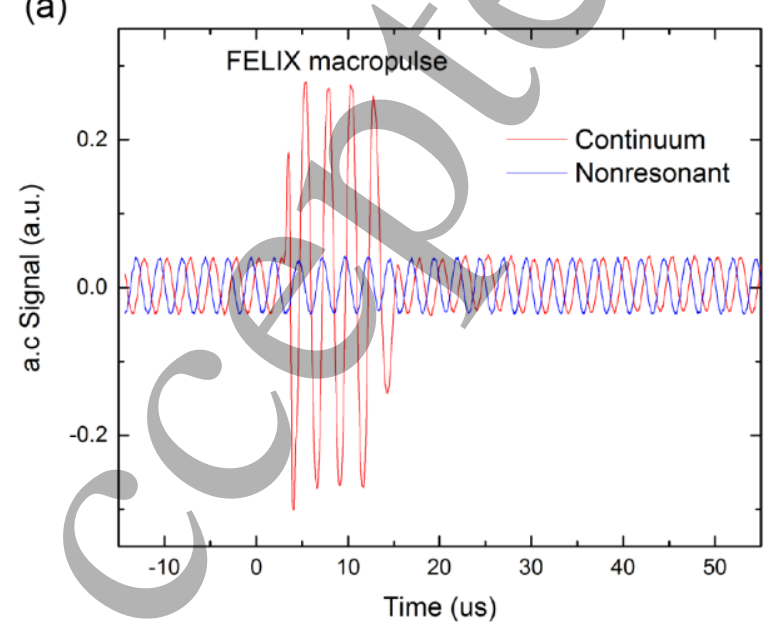

(b)

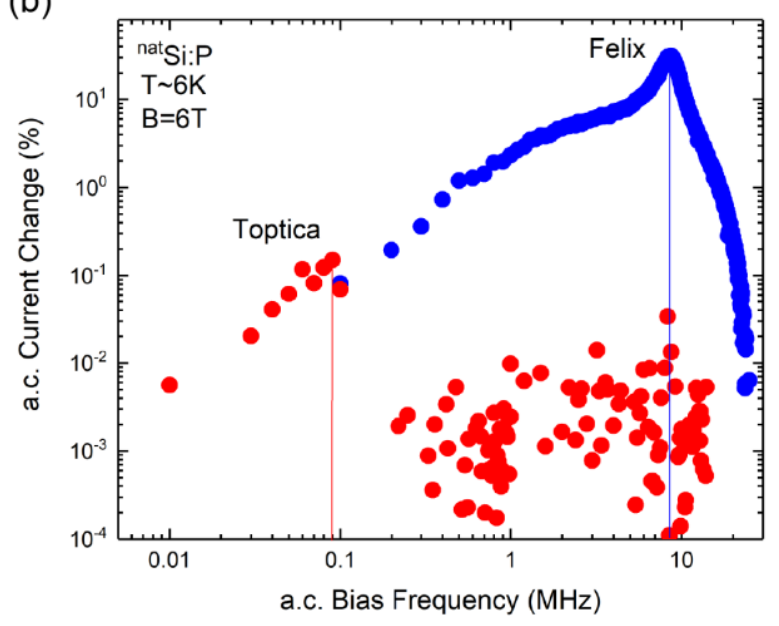


160 Figure 3. (a) Typical a.c. contactless photocurrent signal at $400 \mathrm{kHz}$ ac bias frequency, showing 161 the effect of the arrival of the FELIX pulse. Two FELIX wavelength settings are shown, with non162 resonant excitation and excitation to the continuum. (b) Frequency response of the circuit to the near-IR laser without FELIX (red) and FELIX tuned to the continuum without the near-IR laser (blue) at $B=6 \mathrm{~T}$. Note the peak frequency and also the magnitude of response for Near-IR excitation is almost two orders of magnitude smaller than for the FELIX light, since it is an indirect transition and a weak intensity as a probe.

When FELIX is incident but not tuned to any absorption line, the a.c. photocurrent signal is unaffected. When FELIX is tuned to the 1s-continuum, a large change in photocurrent is observed (Fig 3a,b). When the FELIX pulse is resonant with the 1s-2p transition, it also produces some free electrons due to leakage by thermal excitation from the excited state. For our experimental conditions this number is much larger than the number of electrons generated by the $\mathrm{D}^{0} \mathrm{X}$ process (because the $\mathrm{D}^{0} \mathrm{X}$ absorption is indirect and because the near-IR laser is a deliberately weak probe). The number of electrons generated when FELIX excites directly to the continuum is larger still, and increasing the conductivity, reducing the time constant, and the peak response increases in frequency (Fig $3 b$ ). The short pulse of photocurrent is visible at the moment of the FELIX pulse. This photo-thermal population relaxes due to recombination with the ionized donors, and the number of recombination events per unit time is $\sigma v n_{e} n_{i}$, where $\sigma$ is the capture cross-section, $v$ the electron velocity, $n_{e}$, the number of free electrons and $n_{i}$ the number of ionized donors. For overall neutrality $n_{e}=n_{i}$ so the number of events per unit time is proportional to $n_{e}^{2}$, and the rate per electron is proportional to $n_{e}$, which means that the rate is very fast, at least initially. There is some residual population change many microseconds after the pulse and some residual ringing of the external circuit (Fig 3), but the system response due to FELIX without the near-IR laser is at much higher frequencies than the optimum $\mathrm{D}^{0} \mathrm{X}$ response.

The photo-response is only influenced by FELIX when it is resonant with the Rydberg transitions, as shown by the photon energy dependence (Fig 4). The lines are broad due to the band-width of the laser, and there are some holes in the spectrum due to water vapour in the beam path. The theoretical transition energies for our sample and field orientation were calculated using the Lanczos method [8] and shown in Fig 4. 


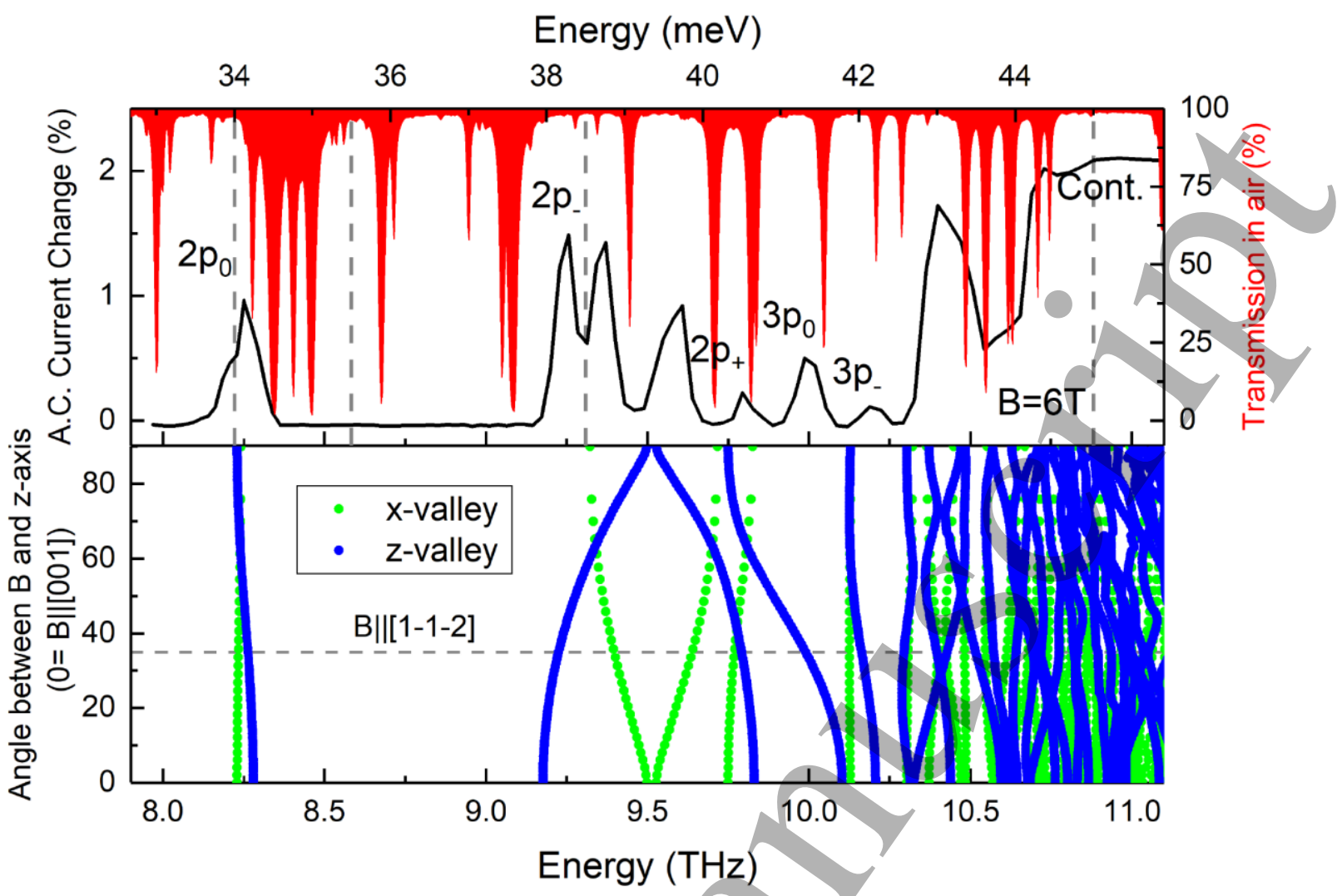

192

193

194

195

196

197

198

199

200

201

202

203

204

205

206

207

208

209

210

211

212

213

214

215

Figure 4. Photo-thermal ionization spectrum in the top panel (determined from the r.m.s. photocurrent under fixed a.c. bias) sweeping the photon energy of the FELIX laser obtained from traces like Fig 3a, at $B=6 \mathrm{~T}$ and $T=6 \mathrm{~K}$ (with the near-IR laser off). The orbital transition energy levels are labelled. The red curve is the experimental transmission of air clearly indicating water vapour absorption lines affecting parts of FEL scan. For the optical pumping experiments below FELIX photon energy was fixed at $34 \mathrm{meV}\left(2 \mathrm{p}_{0}\right), 35.5 \mathrm{meV}$ (non-resonant), $38.5 \mathrm{meV}$ (2p.), and $45 \mathrm{meV}$ (continuum) shown with vertical dashed lines. The bottom panel shows the calculated field direction dependence [8] of orbital transition energies for a [110] sample with $B=6 \mathrm{~T}$. The $y$ axis shows the angle for $B$ in the sample plane for the Voigt geometry; the horizontal dashed line indicates the orientation of the sample used in the experiment. Blue lines: show transitions for the valleys that have their axis along $B$ when $B$ is along [001] (i.e. $\theta=0$ ); green points valleys with axis perpendicular to $B$ when $\theta=0$.

We set the magnetic field to $6 \mathrm{~T}$ and the temperature to $6 \mathrm{~K}$. The $\mathrm{D}^{0} \mathrm{X}$ spectrum was measured: with FELIX blocked; with FELIX tuned to resonance with the $1 \mathrm{~s}-2 \mathrm{p}_{0}$ transition; the $1 \mathrm{~s}-2 \mathrm{p}_{\text {-; }}$ an off-resonance wavelength in between these two; and to a high photon energy for direct excitation to the continuum. Transients like the one shown in Fig 3 a were recorded for postprocessing: we took the r.m.s. amplitude of the photoresponse determined in three "boxcar" windows of width $150 \mu$ s starting at $50 \mu$ s and $150 \mu$ s after FELIX, and $5 \mu$ s before FELIX. The repetition time for the FELIX pulses is $100 \mathrm{~ms}$, so the results for $5 \mu$ s before FELIX also gives an indication of the residual population $100 \mathrm{~ms}$ after FELIX. Examples are shown in Fig 5. The additional noise in Fig 5b is simply due to the FELIX pulse-to-pulse intensity jitter. 


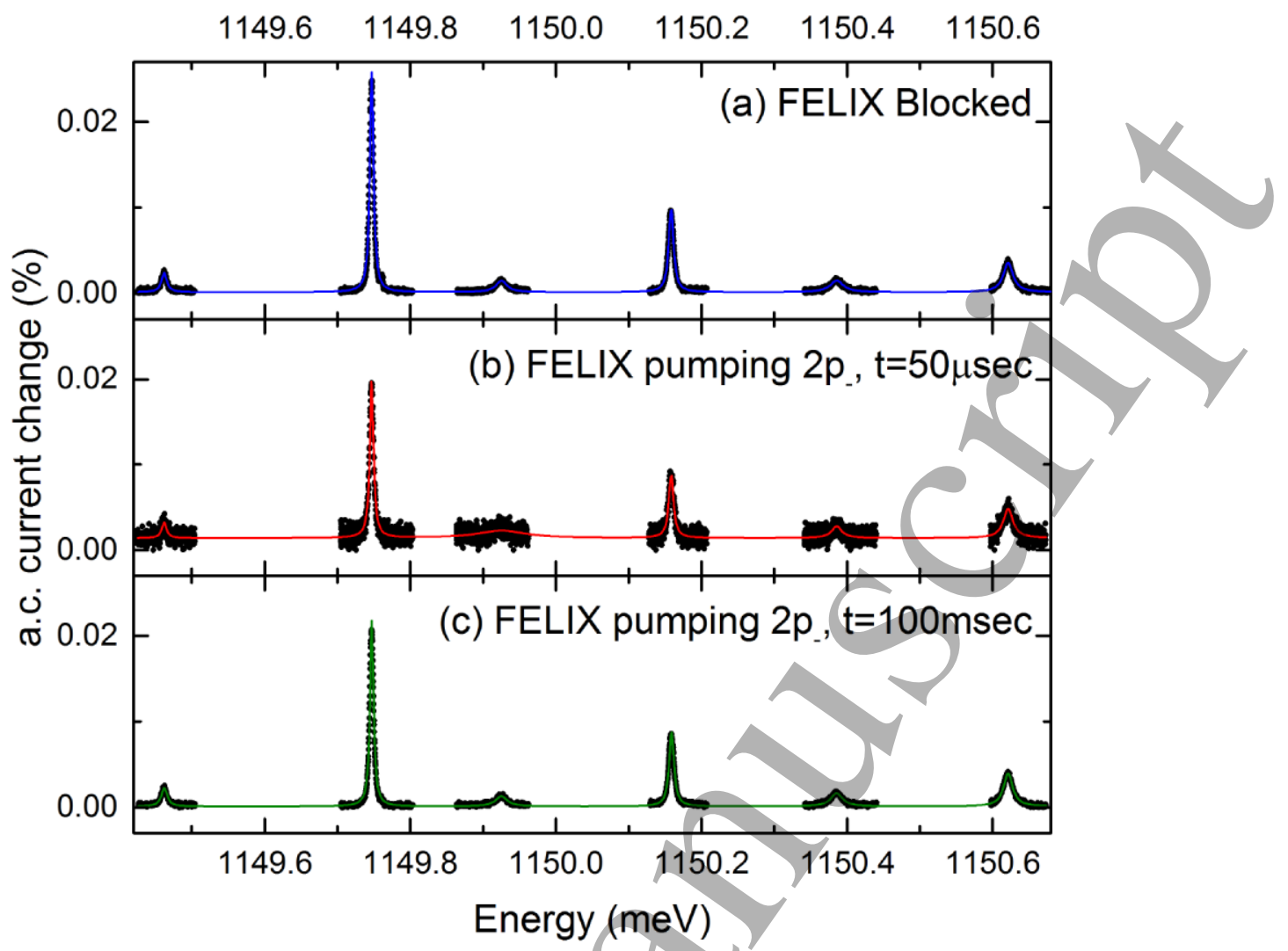

240 Fig $6 \mathrm{~b}$ shows the total population $P(t)=u(t)+d(t)$ and the polarization of the starting state $241 S(t)=(d(t)-u(t)) /(u(t)+d(t))$. It is clear that there is an excitation dependent effect on the

Figure 5. Photoconductivity spectra obtained at $T=6 \mathrm{~K}$ and $B=6 \mathrm{~T}$ for three different conditions of FELIX excitation. (a) FELIX was blocked (same as Fig 1b). (b) The FELIX wavelength was tuned to pump the $1 s \rightarrow 2 p_{-}$transition, and the a.c. photoconductivity amplitude was collected by taking the r.m.s. over a boxcar window at $50 \mu \mathrm{s}$. (c) as (b) for a boxcar at $100 \mathrm{~ms}$ after FELIX macro pulse.

\section{Discussion}

It is clear from the examples in Fig 5 that there is very little effect of FELIX on the relative $\mathrm{D}^{0} \mathrm{X}$ line strengths but there is an obvious overall intensity drop shown in Fig $5 b$ just after FELIX excitation due to elimination/ionization of $\mathrm{D}^{0} \mathrm{X}$ states, their recovery is in ms time scales [1]. In order to quantify the effect of FELIX, we determined the area under each line from a Voigt profile fit, and summed the areas, $L$, of lines $1,3 \& 5$ corresponding to $u=L_{1}+L_{3}+L_{5}$ the population in the starting state $m_{e}=+1 / 2$, and $2,4 \& 6$ corresponding to $d=L_{2}+L_{4}+L_{6}$, for $m_{e}=-$ 1/2. All values for $u$ and $d$ were normalized with the total area under all lines with FELIX blocked, $P_{0}$, and the results $u(t) / P_{0}$ and $d(t) / P_{0}$ for the three time windows and 4 different FELIX wavelengths are shown in Fig 6a. The solid horizontal line indicates the population of the down spin without any FELIX excitation, and the dashed line similarly for the up spin. The down spin polarization decreases under resonant FELIX pumping, and the decrease is strongest immediately after FELIX when pumping to the continuum, and is weaker for excitation between bound states. The decrease is small at very long times after the FELIX pulse. For the up spin there is also a decrease, indicating that the total population of neutral donors drops due to the FELIX pump pulse. 
population, $P(t)$, shortly after the FELIX pulse, but almost no effect on the polarization, $S(t)$

243 for any FELIX excitation condition.

(a)

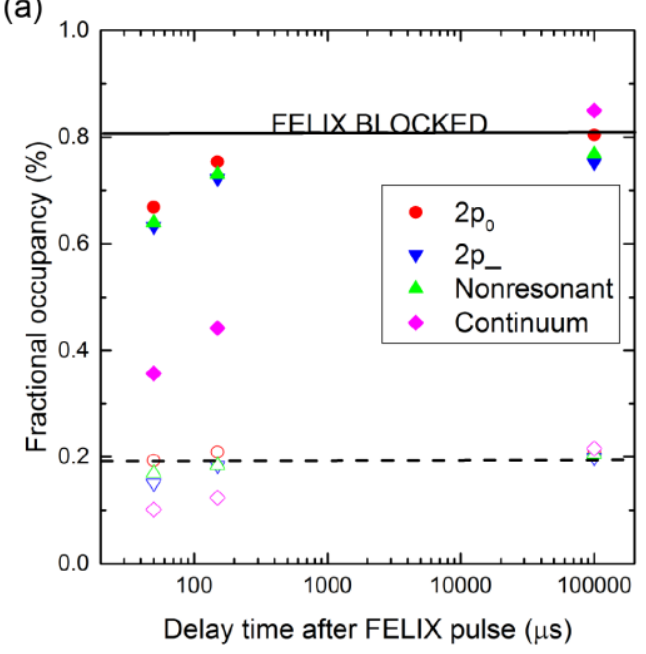

(b)

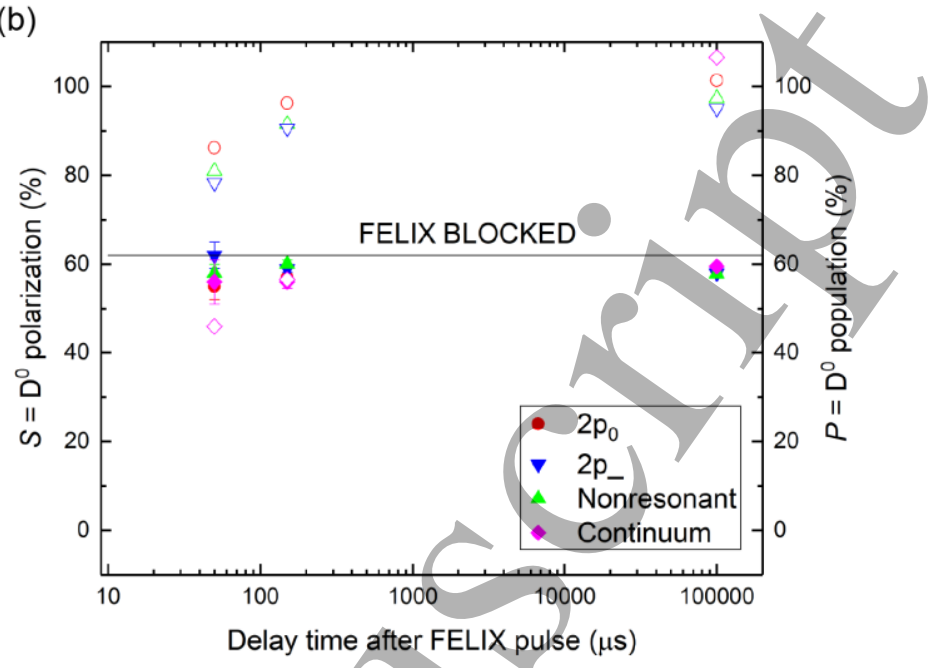

245

246

247

248

249

250

251

252

253

254

255

256

257

258

259

260

261

262

263

264

265

266

267

268

269

270

271

272

273

274

275

276

277

278 indeed low, due to the small matrix element for indirect exciton generation in silicon, and 279 because the near-IR laser beam is deliberately weak. This simply has the effect of making the

different FELIX wavelengths, resonant with bound states $2 p_{0}$ and $2 p_{-}$or the continuum, and non-resonant. (a) The up and down spin populations, $u=\left(L_{1}+L_{3}+L_{5}\right) / P_{0}$ and $d=\left(L_{2}+L_{4}+L_{6}\right) / P_{0}$, where $L_{i}$ is the area under line i from Fig 5 , and normalization was performed using the total area under all lines when FELIX was blocked, $\boldsymbol{P}_{0}$, (open and closed symbols respectively) as a function of time, $t$, after the FELIX pulse. The solid line indicates the value of the thermal downspin population, $d_{0}$, when FELIX was blocked, and the dashed line shows the corresponding $u_{0}$. (b) Time dynamics of the total population (open symbols) $P=u+d$ and spin polarisation (solid symbols) $S=(u-d) /(u+d)$ of the starting states, using the values of $u$ and $d$ from (a). The solid line indicates the background thermal polarization value of $S_{0}=\left(u_{0}-d_{0}\right) /\left(u_{0}+d_{0}\right)$ when FELIX was blocked using the values of $u$ and $d$ from the horizontal lines in (a) (and obviously the corresponding thermal background population $P_{0}=1$ by definition of the normalization).

One should bear in mind that the spin relaxation lifetime within the $\mathrm{D}^{0}$ ground state is well known to be approximately a few hundred milliseconds at the temperature used in the experiment (6K) [5], so within the time-scale $100-200 \mu$ s there is no relaxation, and if the excitation/relaxation produced a significant spin polarization change this ought still to be visible.

The $\mathrm{D}^{0} \mathrm{X}$ creation process is indirect, and therefore rather slow, so it might be suggested that the readout measurement has detected the spin population after all re-equilibration has taken place. The process is $\mathrm{D}^{0}+h v \rightarrow \mathrm{D}^{0} \mathrm{X} \rightarrow \mathrm{D}^{+}+\mathrm{e}^{-}$and the speed of the second step, the Auger decay, may be estimated from the line-widths, Fig 2. In our sample, the lines are homogeneously broadened apart from line 2, and the widths rise with the excited state energy (Fig 2c). This is because the higher energy excited states relax to the lowest energy $m_{h}=-3 / 2$ hole state (the final state of line 2) using phonons of whose density of states increases with transition energy. The $m_{h}=+3 / 2$ state (line 5) has a full width half maximum of $0.032 \mathrm{meV}(=7.74 \mathrm{GHz})$ whose inverse is $T_{l}=21 \mathrm{ps}$. Line 2 is inhomogeneously broadened in our natural silicon sample, but its width has been measured in ${ }^{28} \mathrm{Si}$ and found to be $0.5 \mathrm{neV}(=1.2 \mathrm{MHz})$ giving an inverse of $160 \mathrm{~ns}$. Even this very long time is still much shorter than the spin-relaxation time-scales of interest here. Therefore, the decay part of the process is not limiting, and may be considered instantaneous after the excitation. Consider now the excitation step, i.e. the photon absorption, whose rate is 
probability low that any given $\mathrm{D}^{0}$ atom undergoes the transition, and means that the excitation provides a small and continuous readout sampling. Given that the subsequent Auger relaxation is instantaneous, and the circuit response time is clearly fast enough to resolve the ac bias at $10 \mathrm{MHz}$, the low speed of the $\mathrm{D}^{0} \mathrm{X}$ generation is irrelevant.

Finally, it is also important to establish the strength of the FELIX pumping, since negligible spin relaxation would occur if the pumping were negligible. Note that the total $\mathrm{D}^{0}$ population falls very significantly shortly after the FELIX pulse when pumping directly into the continuum (Fig 5b) and yet the induced polarization change is a few percent at most. (The low change in spin polarization in this case is not surprising, since we might expect that fast spin relaxation occurs in the conduction band). The cross-section for excitation to $1 \mathrm{~s}-2 \mathrm{p}$ - is much larger than for excitation to the continuum [18]. Therefore, even though the photo-conductivity (Fig 4) is smaller because only a small fraction of excited donor electrons is lost from the $2 \mathrm{p}$ - state to the conduction band, an even greater fraction of the population is actually excited when FELIX is resonant with $1 \mathrm{~s}$ to $2 \mathrm{p}$ - than with $1 \mathrm{~s}$ to the continuum. Since the recombination and relaxation following orbital excitation is short $[10,11]$ by comparison with the micropulse repetition time, and there are 100 micropulses in the macropulse, each donor undergoes dozens of excitation events per macropulse. As mentioned above the total spin change after the macropulse is a few percent at most (Fig 6b).

As mentioned in the introduction, there is negligible spin-orbit coupling so the FELIX pump pulse does not affect the spin. It may be that other experiments in which the excitation does not preserve spin (and leaves the polarization in a non-equilibrium situation) would produce different results. The experiment was performed in a substantial magnetic field to prepare the spin polarization and allow easy readout with the $\mathrm{D}^{0} \mathrm{X}$ mechanism, and it would be interesting to investigate the spin preservation at low/zero field [6].

\section{Conclusion}

307 We have tested the relaxation of electron spin for Rydberg excitation of neutral donors using 308 intense THz pulses from the Free Electron Laser FELIX. The results show that resonant 309 excitation to higher Rydberg orbital states followed by phonon relaxation preserves the spin. 310 There is a small loss of population during the excitation cycle due to thermal ionization from 311 the excited state, which could be eliminated by reducing the temperature. Si:P is a possible 312 qubit for solid state quantum computing, and one important gate scheme [4] makes use of THz 313 absorption transitions to excited states as a means to control the interaction between 314 neighbours. The fact the spin is preserved during the excitation and relaxation cycle means that 315 errors caused by unintentional phonon relaxation does not further degrade the quantum gate, 316 and makes the qubit immediately available for subsequent cycles. This could be important for 317 a variety of qubit, quantum sensors and other quantum device applications, where $\mathrm{THz}$ 318 excitations initiate either a coupling or a readout sequence [12].

\section{Acknowledgments}

320 We acknowledge financial support from the UK Engineering and Physical Sciences Research 321 Council (ADDRFSS, Grant No. EP/M009564/1). The work at Nijmegen was performed as part 322 of the research programme of the Stichting voor Fundamenteel Onderzoek der Materie (FOM), 323 which is financially supported by the Nederlandse Organisatie voor Wetenschappelijk 324 Onderzoek (NWO). K.S. would like to thank T. Toonen for his help with the cryostat. 
326 [1] Steger, M., Saeedi, K., Thewalt, M. L. W., Morton, J. J. L., Riemann, H., Abrosimov, N.

327 V., Becker, P., Pohl, H.-J., Quantum Information Storage for over 180s Using Donor Spins in $a^{28} \mathrm{Si}$ "Semiconductor Vacuum", Science 336, 1280-1283 (2012).

329 [2] Karaiskaj, D., inventor; University of South Florida, assignee. Silicon-based atomic clocks. 330 United States Patent US 8,816,784 B1. Aug. 26. (2014).

331 [3] Saeedi, K., Szech, M., Dluhy, P., Salvail, J.Z., Morse, K.J., Riemann, H., Abrosimov, N.V.,

332 Nötzel, N., Litvinenko, K.L., Murdin, B.N., Thewalt, M.L.W., Optical pumping and readout

333 of bismuth hyperfine states in silicon for atomic clock applications, Scientific Reports 5, 10493

334 (2015).

335 [4] Stoneham, A. M., Fisher, A. J. \& Greenland, P. T., Optically driven silicon-based quantum 336 gates with potential for high-temperature operation, J. Phys. Condens. Matter 15, L447-L451 337 (2003).

338 [5] Tyryshkin, A. M., Tojo, S., Morton, J. J. L., Riemann, H., Abrosimov, N. V., Becker, P., 339 Pohl, H-J., Schenkel, T., Thewalt, M. L. W., Itoh, K. M. \& Lyon, S. A., Electron spin coherence 340 exceeding seconds in high-purity silicon, Nature Materials 11,143-147 (2012).

341 [6] Morse, K. J., Dluhy, P., Huber, J., Salvail, J.Z., Saeedi, K., Riemann, H., Abrosimov, N.V., 342 Becker, P., Pohl, H-J., Simmons, S., Thewalt, M. L. W., Zero-field optical magnetic resonance 343 study of phosphorus donors in 28-silicon, Phys. Rev. B 97, 115205 (2018).

344 [7] Feher, G. \& Gere, E. A., Electron spin resonance experiments on donors in silicon. 2. 345 Electron spin relaxation effects, Phys. Rev. 114, 1245-1256 (1959).

346 [8] Murdin, B. N., Juerong Li, Pang, M.L.Y., Bowyer, E.T., Litvinenko, K.L., Clowes, S.K., 347 Engelkamp, H., Pidgeon, C.R., Galbraith, I., Abrosimov, N.V., Riemann, H., Pavlov, S.G., 348 Hübers, H-W., Murdin, P.G., Si:P as a laboratory analogue for hydrogen on high magnetic 349 field white dwarf stars, Nature Commun. 4, 1469 (2013).

[9] Li, J., Litvinenko, K., Clowes, S.K., Engelkamp, H., Pavlov, S.G., Hübers, H. W., Shuman, 351 V. B., Portsel, L. M., Lodygin, A. N., Astrov, Yu. A., Abrosimov, N. V., Pidgeon, C.R., Fisher, 352 A., Zeng, Z., Niquet, Y.-M., Murdin, B.N. Radii of Rydberg states of isolated silicon donors, 353 Phys. Rev. B 98 (8) 085423 (2018)

354 [10] Vinh, N. Q., Greenland, P. T., Litvinenko, K., Redlich, B., van der Meer, A. F. G., Lynch, 355 S. A., Warner, M., Stoneham, A. M., Aeppli, G., Paul, D. J., Pidgeon, C. R., Murdin, B. N., 356 Silicon as a model ion trap: Time domain measurements of donor Rydberg states, PNAS 105, 357 10649-10653 (2008).

358 [11] Bowyer, E. T., Villis, B. J., Li, J., Litvinenko, K. L., Murdin, B. N., Erfani, M., Matmon, 359 G., Aeppli, G., Ortega, J-M., Prazeres, R., Dong, L., Yu, X., Picosecond dynamics of a silicon 360 donor based terahertz detector device, Appl. Phys. Lett. 105, 021107 (2014).

361 [12] Litvinenko, K. L., Pavloy, S. G., Hübers, H.-W., Abrosimov, N. V., Pidgeon, C. R., 362 Murdin, B. N., Photon assisted tunneling in pairs of silicon donors, Phys. Rev. B 89, 235204 363 (2014).

364 [13] Chick, S., Stavrias, N., Saeedi, K., Redlich, B., Greenland, P. T., Matmon, G., Naftaly, 365 M., Pidgeon, C. R. Aeppli G., Murdin B. N.,Coherent superpositions of three states for 366 phosphorous donors in silicon prepared using $\mathrm{THz}$ radiation, Nature Commun. 8, 16038 367 (2017).

368 [14] Yang, A., Steger, M., Karaiskaj, D., Thewalt, M. L. W., Cardona, M., Itoh, K. M., 369 Riemann, H., Abrosimov, N. V., Churbanov, M. F., Gusev, A. V., Bulanov, A. D., Kaliteevskii, 370 A. K., Godisov, O. N., Becker, P., Pohl, H.-J., Ager, J. W., Haller, E. E., Optical Detection and 371 Ionization of Donors in Specific Electronic and Nuclear Spin States, Phys. Rev. Lett. 97, 372 227401(2006). 
373 [15] Litvinenko, K.L., Li, J., Stavrias, N., Meaney, A.J., Christianen, P.C.M, Engelkamp, H., 374 Homewood, K.P., Pidgeon, C.R., Murdin, B.N., Quadratic Zeeman effect used for state-radius 375 determination in neutral donors and donor bound excitons in Si:P, Semicond. Sci. Technol. 37631045007 (2016).

377 [16] Knippels, G. M. H., Yan, X., MacLeod, A. M., Gillespie, W. A., Yasumoto, M., Oepts, 378 D., van der Meer, A. F. G., Generation and Complete Electric-Field Characterization of 379 Intense Ultrashort Tunable Far-Infrared Laser Pulses, Phys. Rev. Lett. 83, 1578, (1999).

380 [17] Hasegawa, H., Spin-Lattice Relaxation of Shallow Donor States in Ge and Si through a 381 Direct Phonon Process, Phys. Rev. 118, 1523 (1960).

382 [18] Pajot, B., Clerjaud, B., Optical Absorption of Impurities and Defects in Semiconducting 383 Crystals, book in Springer Series in Solid-State Sciences (2013). 